\title{
Voltage Instability Analysis Using Mipower
}

\author{
${ }^{1}$ REGATI PRUTHWI TEJA, ${ }^{2}$ KUDURUPAKA VARUN KRISHNA, ${ }^{3}$ KOKKONDA VARUN, \\ ${ }^{4}$ Dr. C BHARGAVA
}

\author{
${ }^{1,2,3}$ B. Tech, ${ }^{4}$ HOD, EEE Dept, SNIST, Hyderabad, India. \\ 17pruthwiregati@gmail.com, ${ }^{2}$ kudurupakavarunkrishna@gmail.com, ${ }^{3}$ kokkondavarun@gmail.com
}

ABSTRACT - Load Flow Analysis helps in error free operation of power system and also useful in forecasting the required equipment for expansion of the system. By forecasting the magnitude of the supply required along with effects caused by single or multiple defects in the system and calculating the magnitude of errors, it is very easy to compensate them using various techniques with minimum cost and effort. It means before installation the favorable sites and size of the infrastructure used are determined to maintain the power factor in the system. Here Power Flow Analysis is performed using Newton Raphson method. This method is used in solving power flow studies of various number of busesunder various conditions. In any network there will be undesired rise or drop or dissipation of voltage. Voltage instability decreases the efficiency of the system and also damages the equipment used. Hence voltage instability analysis is performed and magnitude of the instability is calculated and compensated using various techniques. Here we performed Load Flow Analysis on a 5bus system and Voltage Instability Analysis is also performed to the same with necessary outputs.[7]

KEYWORDS: Newton Raphson Method - Load Flow Analysis, Voltage Instability Analysis, MIPOWER version 9.

\section{INTRODUCTION}

The inability of a system to remain in equilibrium at all the buses whenever subjected to a disturbance called voltage instability. Now a days voltage instability is observed in the system due to lack of co-ordination between generation unit and load unit which results burden on the system. Sometimes the voltages in the system are uncontrollable and may lead to voltage collapse. In some cases the sudden changes in the voltage is undetectable and the effect cannot be stopped. These effects can be studied only after the voltage collapse in order to prevent them in future. The change in load characteristics, increased number of interconnections etc., result in the voltage instability. Use of appliances also depend upon seasons, in summer all the appliances are used more than other seasons which may not result in severe damage but can cause voltage instability and power factor problems. This clearly says that uneven demand for the load also causes voltage instability. Reactive power limit of the generators, long transmission lines also results in voltage instability.[2][8]

COUNTERMEASURES FOR VOLTAGE INSTABILITY [8]

- Under load tap changers

- Reactive compensation devices

- Automatic voltage regulators

- Load shedding etc.,

\section{OBJECTIVES}

First a system of buses is designed and simulated in order to forecast the size and rating of the equipment to be used for construction of a new system or extension of the existing system. All the changes in parameters are observed at different conditions. The simulation can be performed on various softwares like MIPOWER, MATLAB.

\section{MIPOWER}

MIPOWER is a simulation software managed by PRDC Banglore. It is designed by a power systems engineer Dr. Nagrath. The current version of Mipower is 9.0. It is a power systems network analysis package which runs on windows. It is user-friendly and convenient compared to other simulation software.

Accuracy and tolerance is high in MIPOWER. It includes a windows based graphical user interface with centralized database.[3]

Applications of MIPOWER:

Steady State Analysis, Stability Assessment, Transient Studies, Security Monitoring, Assessment and Control,Protection Co-Ordination, Planning Studies,Mipower Utilities.[3]

\section{SOLVING VIA USING MIPOWER}

Open Power system network editor tab and select configure database. Give a name to the file and save it. Now draw the circuit step by step entering the details of elements required. [2]

\section{QUESTION DESCRIPTION}

Perform load flow analysis and voltage instability analysis on a 5 bus power system network with bus voltages $11 \mathrm{kv}$ 
with the following bus, transmission line, generator and load data.[6]

\begin{tabular}{|l|l|l|l|l|l|}
\hline $\begin{array}{l}\text { Bus } \\
\text { no. }\end{array}$ & $\begin{array}{l}\text { Bus } \\
\text { voltage }\end{array}$ & $\begin{array}{l}\text { Generator } \\
(\mathrm{MW})\end{array}$ & $\begin{array}{l}\text { Generation } \\
(\text { MVAR) }\end{array}$ & $\begin{array}{l}\text { Load } \\
(\mathrm{MW})\end{array}$ & $\begin{array}{l}\text { Load } \\
\text { (MVAR) }\end{array}$ \\
\hline 1 & $1+\mathrm{j} 0$ & 0 & 0 & 0 & 0 \\
\hline 2 & $1+\mathrm{j} 0$ & 40 & 30 & 20 & 10 \\
\hline 3 & $1+\mathrm{j} 0$ & 0 & 0 & 45 & 15 \\
\hline 4 & $1+\mathrm{j} 0$ & 0 & 0 & 40 & 5 \\
\hline 5 & $1+\mathrm{j} 0$ & 0 & 0 & 60 & 10 \\
\hline
\end{tabular}

All the p.u values are of 100MVA impedance and line charging for the system.

\begin{tabular}{|l|l|l|}
\hline Bus & Impedance & Line Charging \\
\hline $1-2$ & $0.02+\mathrm{j} 0.06$ & $\mathrm{j} 0.030$ \\
\hline $1-3$ & $0.08+\mathrm{j} 0.24$ & $\mathrm{j} 0.025$ \\
\hline $2-3$ & $0.06+\mathrm{j} 0.18$ & $\mathrm{j} 0.020$ \\
\hline
\end{tabular}

\begin{tabular}{|l|l|l|}
\hline $2-4$ & $0.06+\mathrm{j} 0.18$ & $\mathrm{j} 0.020$ \\
\hline $2-5$ & $0.04+\mathrm{j} 0.12$ & $\mathrm{j} 0.015$ \\
\hline $3-4$ & $0.01+\mathrm{j} 0.03$ & $\mathrm{j} 0.01$ \\
\hline $4-5$ & $0.08+\mathrm{j} 0.24$ & $\mathrm{j} 0.025$ \\
\hline
\end{tabular}

Open Mipower software and open Power System Network Editor. First draw the buses one after one and feed the element details.

Similarly enter the details of generators and loads.

Now solve load flow analysis using any of the four methods. [4]

Then solve voltage instability analysis and save the results. [1]

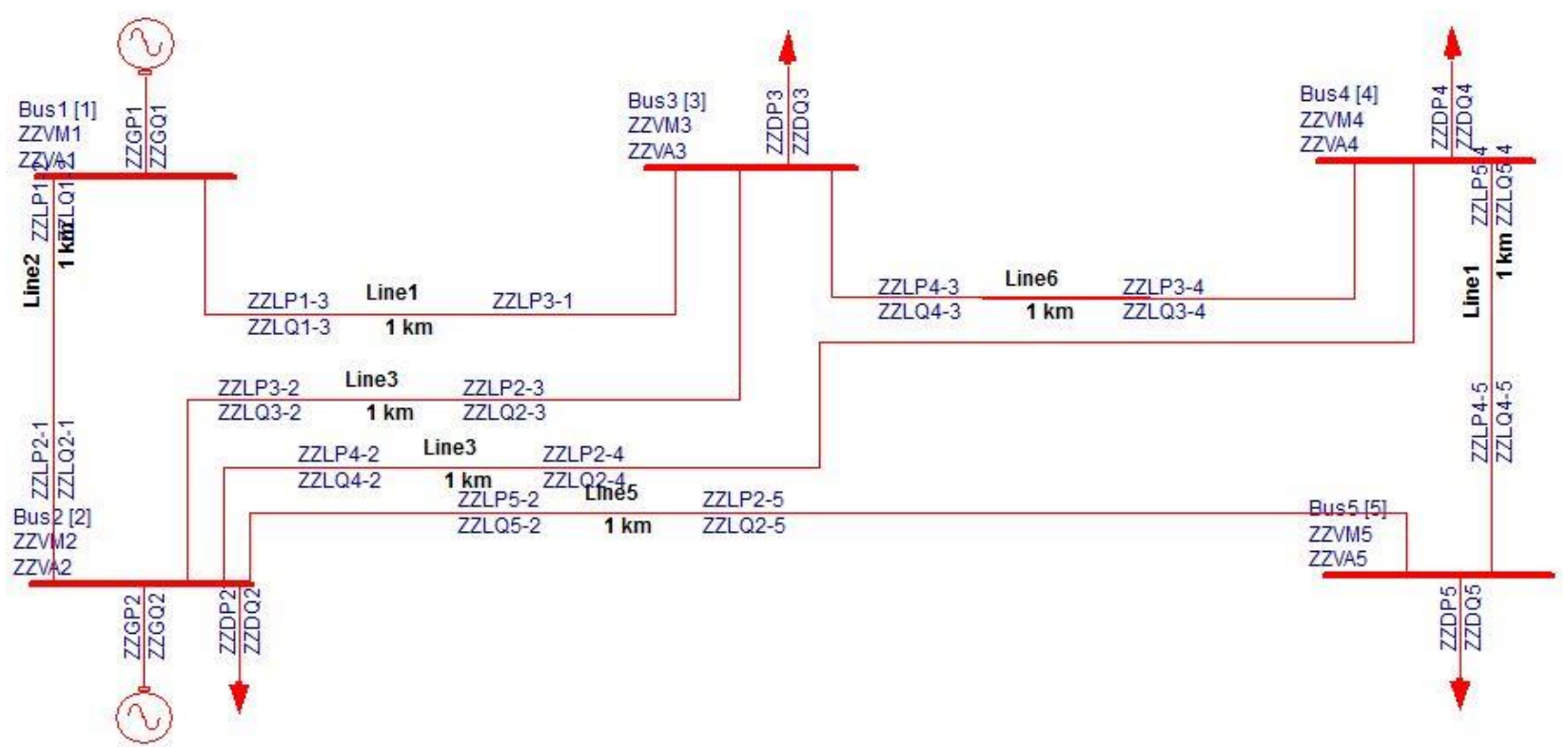

\section{OUTPUT}

\section{LOAD FLOW ANALYSIS USING NEWTON RAPHSON} METHOD

Date and Time : Mon Mar 02 14:52:33 2020

\section{LOAD FLOW BY NEWTON RAPHSON METHOD}

CASE NO : 1 CONTINGENCY : 0 SCHEDULE NO : 0 CONTINGENCY NAME : Base Case RATING CONSIDERED : NOMINAL

\section{VERSION NUMBER : 8.2}

$\% \%$ First Power System Network

Largest Bus Number Used : 5

Actual Number of Buses $\quad: 5$

Number of 2 Wind. Transformers : 0 Number of 3 Wind.

Transformers : 0

Number of Transmission Lines : 7 Number of Series Reactors 0

Number of Series Capacitors : $\quad 0 \quad$ Number of Circuit Breakers $\quad: \quad 0$ Number of Shunt Reactors $\quad: \quad 0$ Number of Shunt Capacitors : 0
Number of Shunt Impedances $\quad: \quad 0 \quad$ Number of Generators $\quad$ : 2 Number of Loads $\quad: \quad 4$ Number of Load Characteristics : 0 Number of Under Frequency Relay: 0 Number of Gen.Capability Curves: 0

Number of Filters $\quad: \quad 0 \quad$ Number of Tie Line Schedules : 0

Number of Convertors $\quad: \quad 0 \quad$ Number of dc Links $\quad: 0$ Number of Shunt Connected Facts: 0 Power Forced Lines 0

Number of TCSC Connected $\quad: \quad 0 \quad$ Number of SPS Connected 0

Number of UPFC Connected $\quad: \quad 0 \quad$ Number of Wind Generators 0

Number of wtg Curves $\quad: \quad 0 \quad$ Number of wtg Detailed Curves : 0

Number of solar plants $\quad: 0$

Load Flow With Newton Raphson Method : 6

Number of Zones $\quad: 1$

Print Option $\quad$ : 3 - Both Data and Results Print

Plot Option : 1 - Plotting with p.u. Voltage

No Frequency Dependent Load Flow, Control Option: 0

Base MVA $\quad: 100.0$ 
Nominal System Frequency (Hz)

Frequency Deviation $(\mathrm{Hz})$

Flows in MW and MVAr, Option

Slack Bus

Transformer Tap Control Option

Q Checking Limit (Enabled)

Real Power Tolerance (p.u.)

Reactive Power Tolerance (p.u.)

Maximum Number of Iterations

Bus Voltage Below Which Load Model is Changed : 0.75000

Circuit Breaker Resistance (p.u.) : $\quad$ : 0.00000

Circuit Breaker Reactance (p.u.) : $\quad: 0.00010$

Transformer R/X Ratio $\quad: 0.05000$

Annual Percentage Interest Charges $\quad: \quad 15.000$

Annual Percent Operation \& Maintenance Charges : 4.000

Life of Equipment in Years $\quad: 20.000$

Energy Unit Charge (KWH) $\quad: \quad 2.500$ Rs

Loss Load Factor $\quad: 0.300$

Cost Per MVAr in Lakhs $\quad$ : $5.000 \mathrm{Rs}$

ZONE WISE MULTIPLICATION FACTORS

ZONE P LOAD Q LOAD P GEN Q GEN SH REACT SH CAP C LOAD

$\begin{array}{llllllll}0 & 1.000 & 1.000 & 1.000 & 1.000 & 1.000 & 1.000 & 1.000\end{array}$

$\begin{array}{llllllll}1 & 1.000 & 1.000 & 1.000 & 1.000 & 1.000 & 1.000 & 1.000\end{array}$

BUS DATA

BUS NO. AREA ZONE BUS kV VMIN(p.u.) VMAX(p.u.) NAME

$\begin{array}{lllllll}1 & 1 & 1 & 11.000 & 0.950 & 1.050 & \text { Bus1 } \\ 2 & 1 & 1 & 11.000 & 0.950 & 1.050 & \text { Bus2 } \\ 3 & 1 & 1 & 11.000 & 0.950 & 1.050 & \text { Bus3 } \\ 4 & 1 & 1 & 11.000 & 0.950 & 1.050 & \text { Bus4 } \\ 5 & 1 & 1 & 11.000 & 0.950 & 1.050 & \text { Bus5 }\end{array}$

\section{TRANSMISSION LINE DATA}

$\begin{array}{lllll}\text { STA CKT } & \text { FROM } & \text { FROM } & \text { TO } & \text { TO } \\ \text { LINE PARAMETER }\end{array}$ RATING KMS

NODE NAME* NODE NAME* R(p.u.) X(p.u.) B/2(p.u.)

MVA

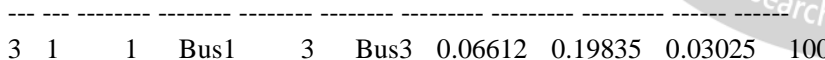

1.00

$\begin{array}{llllllllll}3 & 1 & 1 & \text { Bus1 } & 2 & \text { Bus2 } & 0.01653 & 0.17025 & 0.03630 & 100\end{array}$

1.00

$\begin{array}{llllllllll}3 & 1 & 2 & \text { Bus2 } & 3 & \text { Bus3 } & 0.04959 & 0.14876 & 0.02420 & 100\end{array}$

1.00

$\begin{array}{llllllllll}3 & 1 & 2 & \text { Bus2 } & 4 & \text { Bus4 } & 0.04959 & 0.14876 & 0.02420 & 100\end{array}$

1.00

$\begin{array}{llllllllll}3 & 1 & 2 & \text { Bus2 } & 5 & \text { Bus5 } & 0.03306 & 0.09917 & 0.01815 & 100\end{array}$

1.00

$\begin{array}{llllllllll}3 & 1 & 3 & \text { Bus3 } & 4 & \text { Bus4 } & 0.00826 & 0.02479 & 0.01210 & 100\end{array}$

1.00

$\begin{array}{llllllllll}3 & 1 & 4 & \text { Bus4 } & 5 & \text { Bus5 } & 0.06612 & 0.19835 & 0.03025 & 100\end{array}$

1.00

Total Line Charging Susceptance (in p.u.) : 0.35090

Total Line Charging MVAr at 1 p.u. Voltage

$: 35.090$

Number of Lines Opened on Both the Ends

0

Total Line Charging susceptance of Existing Lines (in p.u.) : 0.35090

Total Line Charging MVAr at 1 p.u. Voltage of Existing Lines : 35.090

Total Capacitive Susceptance : 0.00000 p.u. - $0.000 \mathrm{MVAr}$

Total Inductive Susceptance : 0.00000 p.u. - $0.000 \mathrm{MVAr}$
GENERATOR DATA

SI.No* FROM FROM REAL Q-MIN Q-MAX V-SPEC CAP. MVA STAT

NODE NAME*POWER(MW) MVArMVArp.u. CURV RATING

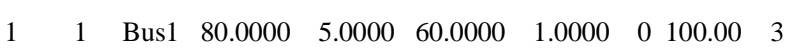

$\begin{array}{llllllllll}2 & 2 & \text { Bus2 } & 80.0000 & 5.0000 & 60.0000 & 1.0000 & 0 & 100.00 & 3\end{array}$

LOAD DATA

Sl.No. FROM FROM REAL REACTIVE COMP COMPENSATING MVAR VALUE CHAR F/V

* NODE NAME* MW MVArMVAr MIN MAX STEP NO. NO

STAT

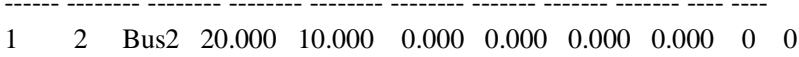

30

$\begin{array}{lllllllllll}2 & 3 & \text { Bus3 } & 45.000 & 15.000 & 0.000 & 0.000 & 0.000 & 0.000 & 0 & 0\end{array}$

30

$\begin{array}{lllllllllll}3 & 4 & \text { Bus4 } & 40.000 & 5.000 & 0.000 & 0.000 & 0.000 & 0.000 & 0 & 0\end{array}$

30

$\begin{array}{lllllllllll}4 & 5 & \text { Bus5 } & 60.000 & 10.000 & 0.000 & 0.000 & 0.000 & 0.000 & 0 & 0\end{array}$

30

Total Specified MW Generation $\quad: 160.00000$

Total Minimum MVAr Limit of Generator : 10.00000

TOTAL Maximum MVAr Limit of Generator : 120.00000

Total Specified MW Load $\quad: 165.00000$ Changed to 165.00000 Total Specified MVAr Load $\quad$ : 40.00000 Changed to 40.00000 Total Specified MVAr Compensation : 0.00000 Changed to 0.00000

TOTAL (Including Out of Service Units)

Total Specified MW Generation $\quad: 160.00000$

TOTAL Minimum MVAr Limit of Generator : 10.00000

Total Maximum MVAr Limit of Generator : 120.00000

Total Specified MW Load $\quad: 165.00000$ Changed to 165.00000

Total Specified MVAr Load $\quad: \quad 40.00000$ Changed to 40.00000

Total Specified MVAr Compensation : 0.00000 Changed to 0.00000

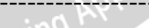

GENERATOR DATA FOR FREQUENCY DEPENDENT LOAD FLOW

SLNO* FROM FROM P-RATE P-MIN P-MAX \%DROOP PARTICI BIAS NODE NAME* MW MWMW FACTOR SETTING $\mathrm{C} 0 \quad \mathrm{C} 1 \quad \mathrm{C} 2$

$\begin{array}{lllllllll}1 & 1 & \text { Bus1 } & 80.000 & 20.0000 & 80.0000 & 4.0000 & 0.0000 & 0.0000\end{array}$ $\begin{array}{llll}0.0000 & 0.0000 & 0.0000\end{array}$

$\begin{array}{lllllllll}2 & 2 & \text { Bus2 } & 40.000 & 20.0000 & 80.0000 & 4.0000 & 0.0000 & 0.0000\end{array}$ $\begin{array}{lll}0.0000 & 0.0000 & 0.0000\end{array}$

Slack bus angle (degrees) : 0.00

TOTAL NUMBER OF ISLANDS IN THE GIVEN SYSTEM $\quad: \quad 1$ TOTAL NUMBER OF ISLANDS HAVING ATLEAST ONE GENERATOR : 1

SLACK BUSES CONSIDERED FOR THE STUDY

ISLAND NO. SLACK BUS NAME SPECIFIED MW

$1 \quad 1 \quad$ Bus1 80.000

Iteration count 0 maxp $0.600000 \operatorname{maxq} 0.083450$ 
Iteration count 1 maxp 0.019533 maxq 0.026128

Iteration count 2 maxp 0.000055 maxq 0.000097

Iteration count 3 maxp 0.000000 maxq 0.000000

Iteration count 4 maxp 0.000000 maxq 0.000000

BUS VOLTAGES AND POWERS

NODE FROM V-MAG ANGLE MW MVAr MW MVArMVAr

NO. NAME p.u. DEGREE GEN GEN LOAD LOAD COMP

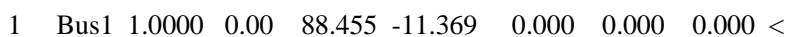

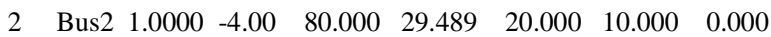

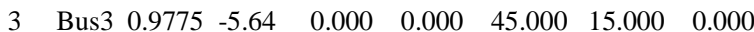

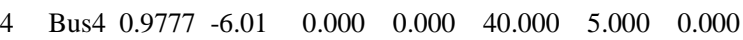

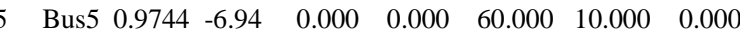

NUMBER OF BUSES EXCEEDING MINIMUM VOLTAGE LIMIT (@ mark):0

NUMBER OF BUSES EXCEEDING MAXIMUM VOLTAGE LIMIT (\# mark) : 0

NUMBER OF GENERATORS EXCEEDING MINIMUM Q LIMIT (<mark) : 1

NUMBER OF GENERATORS EXCEEDING MAXIMUM Q LIMIT (>mark) : 0

LINE FLOWS AND LINE LOSSES

$\begin{array}{llllll}\text { SLNO CS } & \text { FROM } & \text { FROM } & \text { TO } & \text { TO } & \text { FORWARD }\end{array}$ $\begin{array}{lllllll}\text { LOSS } & \% & & & & & \\ \text { NODE } & \text { NAME } & \text { NODE } & \text { NAME } & \text { MW } & \text { MVAr } & \text { MW }\end{array}$ MVAr LOADING

\begin{tabular}{llllllllll}
\hline \multicolumn{1}{c}{1} & 1 & Bus1 & 3 & Bus3 & 47.716 & -5.215 & 1.5085 & -1.3901 \\
$48.0^{\wedge}$ & & & & & & & & \\
$2 \quad 1$ & 1 & Bus1 & 2 & Bus2 & 40.739 & -6.154 & 0.2754 & -4.4236 \\
$41.2^{\wedge}$ & & & & & & & & \\
$3 \quad 1$ & 2 & Bus2 & 3 & Bus3 & 21.540 & 5.773 & 0.2634 & -3.9424 \\
$23.9 \&$ & & & & & & & & \\
$4 \quad 1$ & 2 & Bus2 & 4 & Bus4 & 25.411 & 4.527 & 0.3441 & -3.7008 \\
$27.0^{\wedge}$ & & & & & & & & \\
$5 \quad 1$ & 2 & Bus2 & 5 & Bus5 & 53.513 & 7.458 & 0.9751 & -0.6130 \\
$54.0 \$$ & & & & & & & & \\
61 & 3 & Bus3 & 4 & Bus4 & 22.484 & -9.110 & 0.0492 & -2.1652 \\
$24.8 \&$ & & & & & & & & \\
$7 \quad 1$ & 4 & Bus4 & 5 & Bus5 & 7.502 & -3.717 & 0.0394 & -5.6453 \\
$8.6 \&$ & & & & & & & &
\end{tabular}

! NUMBER OF LINES LOADED BEYOND $125 \% \quad: 0$

@ NUMBER OF LINES LOADED BETWEEN 100\% AND 125\%: 0

\# NUMBER OF LINES LOADED BETWEEN 75\% AND 100\%: 0

\$ NUMBER OF LINES LOADED BETWEEN 50\% AND 75\%: 1

$\wedge$ NUMBER OF LINES LOADED BETWEEN 25\% AND 50\%: 3

\& NUMBER OF LINES LOADED BETWEEN 1\% AND 25\% : 3

* NUMBER OF LINES LOADED BETWEEN $0 \%$ AND $1 \%: 0$

BUSES BETWEEN WHICH ANGLE DIFFERENCE IS > 30 degrees ARE: ZERO

ISLAND FREQUENCY SLACK-BUS CONVERGED(1)

$150.00000 \quad 1 \quad 1$

Summary of results
TOTAL REAL POWER GENERATION (CONVENTIONAL) 168.455 MW

TOTAL REAL POWER INJECTION (-veLOAD) : : $0.000 \mathrm{MW}$ TOTAL REACT. POWER GENERATION (CONVENTIONAL) 18.120 MVAr GENERATION p.f. $\quad: \quad 0.994$ TOTAL REAL POWER GENERATION (WIND) : : $0.000 \mathrm{MW}$ TOTAL REACT. POWER GENERATION (WIND) : $\quad 0.000$ MVAr

TOTAL REAL POWER GENERATION (SOLAR) : $\quad 0.000 \mathrm{MW}$ TOTAL REACT. POWER GENERATION (SOLAR) : : 0.000 MVAr

TOTAL SHUNT REACTOR INJECTION TOTAL SHUNT REACTOR INJECTION TOTAL SHUNT CAPACIT.INJECTION TOTAL SHUNT CAPACIT.INJECTION TOTAL TCSC REACTIVE DRAWL TOTAL SPS REACTIVE DRAWL TOTAL UPFC INJECTION TOTAL SHUNT FACTS INJECTION TOTAL SHUNT FACTS DRAWAL TOTAL REAL POWER LOAD TOTAL REAL POWER DRAWAL (-ve gen.) TOTAL REACTIVE POWER LOAD LOAD p.f. : $\quad 0.972$ TOTAL COMPENSATION AT LOADS TOTAL HVDC REACTIVE POWER

$-0.000 \mathrm{MW}$ $-0.000 \mathrm{MVAr}$ $-0.000 \mathrm{MW}$ $-0.000 \mathrm{MVAr}$ $0.000 \mathrm{MVAr}$ $0.000 \mathrm{MVAr}$ $-0.000 \mathrm{MVAr}$ $0.000 \mathrm{MVAr}$ $0.000 \mathrm{MVAr}$ $165.000 \mathrm{MW}$ $0.000 \mathrm{MW}$ 40.000 MVAr

TOTAL REAL POWER LOSS (AC+DC) $3.455023+0.000000)$ PERCENTAGE REAL LOSS (AC+DC) TOTAL REACTIVE POWER LOSS

\subsection{MVAr $0.000 \mathrm{MVAr}$}

$3.455023 \mathrm{MW}$

\begin{tabular}{|c|c|}
\hline \multicolumn{2}{|c|}{ Zone wise distribution } \\
\hline Description & Zone \# 1 \\
\hline MW generation & 168.4550 \\
\hline MVAr generation & 18.1196 \\
\hline MW wind gen. & 0.0000 \\
\hline MVAr wind gen. & 0.0000 \\
\hline MW solar gen. & 0.0000 \\
\hline MVAr solar gen. & 0.0000 \\
\hline MW load & 165.0000 \\
\hline MVAr load & 40.0000 \\
\hline MVAr compensati & tion $\quad 0.0000$ \\
\hline MW loss & 3.4550 \\
\hline MVAr loss & -21.8804 \\
\hline MVAr - inductive & 0.0000 \\
\hline MVAr - capacitive & e 0.0000 \\
\hline
\end{tabular}

Zone wise export(+ve)/import(-ve)

Zone \# $1 \mathrm{MW} \& \mathrm{MVAr}$

1

Area wise export(+ve)/import(-ve)

Area \# $1 \mathrm{MW} \& \mathrm{MVAr}$

1

Area wise distribution

Description Area \# 1

MW generation 168.4550

MVAr generation $\quad 18.1196$

MW wind gen. $\quad 0.0000$

MVAr wind gen. $\quad 0.0000$

MW solar gen. $\quad 0.0000$

MVAr solar gen. $\quad 0.0000$

MW load $\quad 165.0000$ 
MVAr compensation $\quad 0.0000$

MW loss $\quad 3.4550$

MVAr loss $\quad-21.8804$

MVAr - inductive $\quad 0.0000$

MVAr - capacitive 0.0000

Date and Time : Mon Mar 02 14:52:33 2020

\section{VOLTAGE INSTABILITY ANALYSIS}

Date and Time : Mon Mar 02 15:25:02 2020

VOLTAGE INSTABILITY ANALYSIS

$\mathrm{NO}: 0$

CONTINGENCY NAME : Base Case

VERSION NUMBER : 8.1
MVAr load $\quad 40.0000$

CASE NO : 1 CONTINGENCY : $0 \quad$ SCHEDULE
$3 \quad 1$

1.00

$3 \quad 1$

1.00

31

1.00

$3 \quad 1$

1.00

$3 \quad 1$

1.00

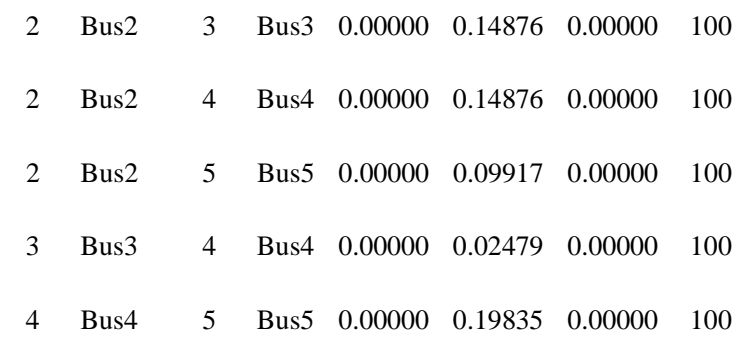

LOAD DATA

Sl.No. FROM FROM REAL REACTIVE COMP COMPENSATING MVAR VALUE CHAR F/V

* NODE NAME* MW MVArMVAr MIN MAX STEP NO. NO.

STAT

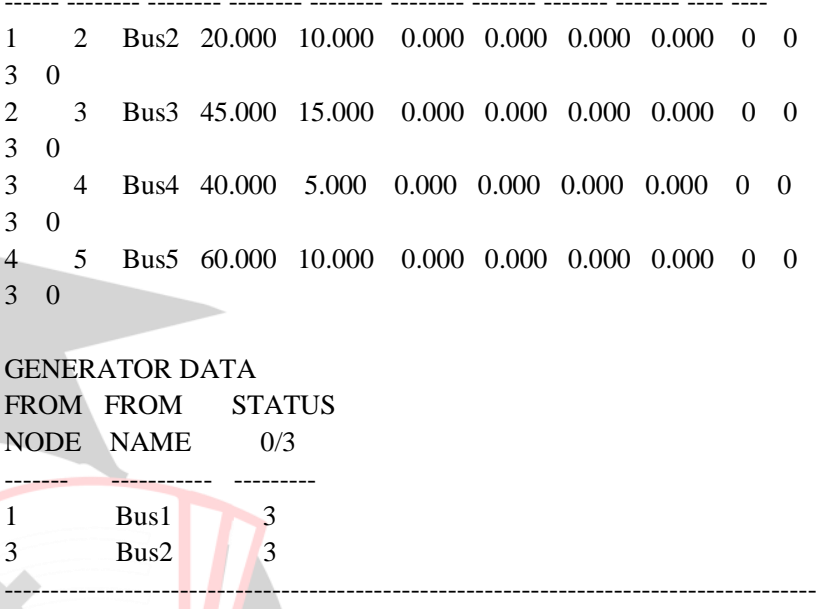

CENTROID VOLTAGE OF GENERATOR BUS VOLTAGES $(1.082234)+(\mathrm{j}-0.004635)$

L INDEX VALUE AND VCPI (Centroid) FOR THE SYSTEM AT GIVEN OPERATING CONDITION

$\begin{array}{lll}\text { NUMBER OF ZONES } & : 1 \\ \text { PRINT OPTION } & : 3 \text { (BOTH DATA AND RESULTS }\end{array}$

PRINT)

PLOT OPTION : 0 (NO PLOT FILE GENERATION)

BASE MVA : 100.000

NOMINAL SYSTEM FREQUENCY: 50.000

CIRCUIT BREAKER RESISTANCE (PU)

CIRCUIT BREAKER REACTANCE (PU)

TRANSFORMER R/X RATIO

BUS DATA

BUS NO. AREA ZONE BUS kV VMIN(p.u.) VMAX(p.u.) NAME

$\begin{array}{lllllll}1 & 1 & 1 & 11.000 & 0.950 & 1.050 & \text { Bus1 } \\ 2 & 1 & 1 & 11.000 & 0.950 & 1.050 & \text { Bus2 } \\ 3 & 1 & 1 & 11.000 & 0.950 & 1.050 & \text { Bus3 } \\ 4 & 1 & 1 & 11.000 & 0.950 & 1.050 & \text { Bus4 } \\ 5 & 1 & 1 & 11.000 & 0.950 & 1.050 & \text { Bus5 }\end{array}$

TRANSMISSION LINE DATA

STACKT FROM FROM TO TO LINE PARAMETER

RATING KMS

NODE NAME* NODE NAME* R(p.u.) X(p.u.) B/2(p.u.)

MVA

$\begin{array}{lrllllllll}3 & 1 & 1 & \text { Bus1 } & 3 & \text { Bus3 } & 0.00000 & 0.19835 & 0.00000 & 100 \\ 1.00 & & & & & & & & \\ 3 & 1 & 1 & \text { Bus1 } & 2 & \text { Bus2 } & 0.00000 & 0.17025 & 0.00000 & 100\end{array}$

SLNO BUSNO NAME VOLT-MAG L-INDEX VCPIcentroid

$\begin{array}{llllll}0.000000 & 1 & 3 & \text { Bus3 } & 0.931822 & 0.102354 \\ 0.000100 & 0.104873 & & & \\ 0.050000 & 2 & 4 & \text { Bus4 } & 0.938109 & 0.105763 \\ & 0.100012 & & & \\ & 3 & 5 & \text { Bus5 } & 0.912197 & 0.190289 \\ \text { NAME } & 0.194873 & & \end{array}$

\section{CONCLUSION}

Load Flow Analysis is used for perfect planning and operation of a power system. This paper indicates the load flow analysis using Newton Raphson method and Voltage Instability Analysis on a 5 bus system. MIPOWER is helpful in easy and accurate solving of power flow studies and results in power and voltage enhancement. The voltage and phase is obtained at each bus along with real and reactive power flowing in each line during the normal conditions and voltage instability. The voltage instability at load bus is determined and noted. [5] 


\section{REFERENCES}

[1] IJESI research paper- "Load Flow and Voltage Instability using MI Power Software" by Vibha Parmar (Asst. Professor), Margi Shah (Junior Research Fellow), Electrical Department, Charusat University, India.

[2] IJERT research paper- "Load Flow Analysis of IEEE 3 bus system by using MIPOWER Software" by Sandeep Kaur (Asst. Professor, EEE Dept), Amarbir Singh (Asst. Professor, Mechanical Dept), Dr. Raja Singh Khela (Director, Jasdev Singh Sandhu Institute of Engg\& Tech - Punjab).

[3] PRDC MIPOWER official page (http://www.prdcinfotech.com/business/softwareengineering-group/softwareproducts/mipower/mipower-applications/).

[4] IJSRP ,Vol.2,Issue 11, Nov. 2012."Load Flow Analysis on IEEE 30 bus System " byDharamjit and D.K. Tanti.

[5] "A novel comparison of Gauss-Seidel and Newton Raphson methods for load flow analysis",IEEE Trans. Power Systems ,vol.10, no.1109, ICIEECT.2017. 17289904, 16-18 March 2017 by Sreemoyee Chatterjee \&Suprovab Mandal.

[6] "Load Flow Analysis with Voltage Sensitive Loads", 2008 Joint International Conference on Power System by C.S. Indulkar.

[7] "Power Flow Management For Grid Stability using TCSC device”, 2018 IEEE PIICON by Jasjeet Singh, Yajvennder Pal Verma.

[8] "A comprehensive review of the voltage stability indices", Renewable and Sustainable Energy Reviews, 2016 by M. Javad, G. Eskandar, k. Amin. 